\title{
Diffusion gravity and its consequences
}

\author{
Zahid Zakir (1) \\ Centre for Theoretical Physics and Astrophysics, Tashkent, Uzbekistan \\ zzakir@qgph.org
}

\begin{abstract}
Diffusion quantum mechanics (DQM), proposed recently (Zakir, 2020-21), describes a conservative diffusion of classical particles in a fluctuating classical scalar field and, in a homogeneous field, derives the formalism of quantum mechanics. In an inhomogeneous scalar field, DQM reproduces gravitation, and in the present paper, the following theory of diffusion gravity and its various consequences are considered. In DQM a part of the energy of the scalar field is transferred to particles as their fluctuation energy ("thermal" energy), appearing as their rest energy (mass). The resulting local decrease in the field's energy density around a macroscopic body generates "thermal" diffusion flux of particles to this region. The properties of this "thermal" part of conservative diffusion are similar to gravitation. A high matter concentration in some region reduces the local energy density of scalar field sufficiently to reduce the local intensity of fluctuations. Due to the conservativity of diffusion, the increments in the drift velocity of particles are cumulative, and "thermal" diffusion acceleration arises, independent on the particle's mass. The world lines become curved, and all processes with particles slowdown, which means time dilation. On hypersurfaces of simultaneity $t=$ const, where the scalar field is defined, effective metrics, connection, and curvature arise. They obey to Einstein's equations following from balance between energies of matter and background scalar field.
\end{abstract}

\section{INTRODUCTION}

The diffusion treatment of quantum mechanics, described in [1], proceeds from the experimental facts that an initially localized ensemble of free microparticles spreads in space (a wave packet spreading) as in a certain frictionless diffusion similar in any inertial frame. It was shown that an ensemble of classical particles interacting with a fluctuating classical scalar field performs the same conservative diffusion, also obeying the principle of relativity. Then, on the basis of this equivalence, diffusion quantum mechanics (DQM) was formulated, which in the uniform scalar field reproduces quantum mechanics and also has new consequences.

In the case of inhomogeneous scalar field there is an analog of thermal diffusion, the properties of which exactly the same as for gravitation. As it was explained in [1] and studied preliminarily in [2], this direct consequence of DQM leads to the diffusion treatment of gravitation, or diffusion gravity. In the present paper, diffusion gravity will be formulated systematically and its consequences for particle physics, astrophysics and cosmology will be studied.

DQM is described in Section 2, in Sections 3-5 the causes for the formation of inhomogeneities in the scalar field and how gravity appears as their consequence are described. In Sections 6-7, the physical restrictions for the formalism of general relativity and the problems with gravitational energy and their solution in diffusion gravity are described.

\section{DIFFUSION TREATMENT OF QUANTUM MECHANICS}

In [1] DQM is formulated as the theory of conservative diffusion of classical particles in the fluctuating classical scalar field. In the conservative diffusion, the probability density $\rho(\mathbf{x}, t)$ and the diffusion flux $\mathbf{j}_{D} \equiv \mathbf{u} \rho$, where $\mathbf{u}(\mathbf{x}, t)$ is the local velocity of diffusion flux, are related, as in any diffusion, by Fick's law $\mathbf{j}_{D}=-D \nabla \rho$. In the case of homogeneous scalar field the diffusion coefficient $D$ is constant, invariant and vanishes at large mass. The correspondence with classical and quantum mechanics gives $D=\hbar / 2 m$ and we have for $\mathbf{u}$ :

$$
\mathbf{u}=-\frac{\hbar}{2 m} \frac{\nabla \rho}{\rho}, \quad \overline{\mathbf{u}}=\int \mathbf{u} \rho d^{3} \mathbf{x}=0, \quad \int \mathbf{u}^{2} \rho d^{3} \mathbf{x} \geq 0 .
$$

The corresponding diffusion momentum is $\mathbf{p}_{\mathbf{u}}=m \mathbf{u}$. The drift velocity $\mathbf{v}(\mathbf{x}, t)$, which is constant for free particles, is similar to the classical velocity and thus the drift momentum $\mathbf{p}_{\mathbf{v}}=m \mathbf{v}$ is related by the drift action function $S(\mathbf{x}, t)$ as $\mathbf{p}_{\mathbf{v}}=\nabla S$.

The Hamilton function $H$ of an ensemble of one particle states in the potential $V$ includes a "thermal" energy of fluctuations $U_{m}$, identifying by the rest energy $U_{m}=m c^{2}$, kinetic energies of drift $(\nabla S)^{2} / 2 m$ and diffusion flux $U_{u}=\mathbf{p}_{\mathbf{u}}^{2} / 2 m$. At omitting terms of order $1 / c^{2}, H$ has the for:

$$
H=\int\left(U_{m}+\frac{1}{2 m}(\nabla S)^{2}+\frac{\hbar^{2}}{8 m}\left(\frac{\nabla \rho}{\rho}\right)^{2}+V\right) \rho d^{3} \mathbf{x} .
$$


Here $\rho$ and $S$ form a canonical pair and canonical equations for them in the phase space $(\rho, S)$ lead to the Hamilton-JacobiMadelung and continuity equations. These two real valued equations are non-linear, but at the canonical transformation $\psi=\rho^{1 / 2} e^{i S / \hbar}$ to the phase space of complex conjugate probability amplitudes $\left(\psi^{*}, \psi\right)$ they form one complex and linear equation, the Schrödinger equation. Thus, DQM reproduces the formalism of quantum mechanics.

Since DQM interprets the rest energy $E_{0}=m c^{2}$ as a "thermal" energy of the random walking massless particles, then those of them that move rectilinearly do not have rest energy (mass) and their drift velocity is equal to the light velocity.

In the presence of gauge fields $A_{\mu}^{(a)}$, the Lagrange function density of the scalar field $\left|D_{\mu} \phi\right|^{2}=\left|\left(\partial_{\mu}-i f^{(a)} A_{\mu}^{(a)} / 2\right) \phi\right|^{2}$, taking into account that $\bar{\phi}=0$ and $\overline{|\phi|^{2}}=\eta^{2}$, leads to the masses of gauge bosons $m^{(a)}=f^{(a)} \eta / 2$, exactly as in the Standard Model. For fermions the interaction term may include linear and quadratic under $\phi$ terms and the latter leads to the mass term.

Thus, a fluctuating part of $\phi$ leads to quantum fluctuations, while its rms - to the particle's mass (rest energy). This new mass generation mechanism requires the fluctuations of the classical scalar field only, while spontaneous symmetry breaking is not necessary. DQM also leads to a new mechanism for low mass of composite particles, which can solve the mass problem in the models of substructure of quarks and leptons.

\section{GRAVITY AS A LOCAL DEFICIT OF FLUCTUATION ENERGY OF SCALAR FIELD}

In DQM particle's fluctuations occur due to energy exchange with the scalar field, while total energy of the system "particle + scalar field" is conserved. As a result, the mean energy of fluctuations, transferred from the scalar field to the particle, decreases the field's energy to the same value. As the result, the energy density of the scalar field near particle $\rho_{\eta^{\prime}}$ becomes less than the energy density at a large distance $\rho_{\eta}$, i.e. $\rho_{\eta^{\prime}}<\rho_{\eta}$.

The influence of one or several microparticles on $\rho_{\eta}$ is insignificant, but a high matter density in a certain region reduces $\rho_{\eta}$ sufficiently to reduce the local fluctuation energy of particles in this region. Really, a decreased energy density $\rho_{\eta^{\prime}}<\rho_{\eta}$ means that $\eta^{2}<\eta^{2}$ and, accordingly, the fluctuation energy of particles in this region also appears decreased. Since a smaller $\rho_{\eta^{\prime}}$ is equivalent to a local "cooling" of the fluctuating background, there appears an analog of the thermodiffusion flux of particles from regions with a higher $\rho_{\eta}$ to this region with a lower $\rho_{\eta^{\prime}}$. As a result, the region with a very high concentration of particles effectively attracts other particles.

Due to the conservativity of diffusion in the scalar field, lack of friction, the velocity of such a "thermodiffusion" flux of particles will increase cumulatively, i.e. here there is a "thermal" diffusion acceleration. This acceleration depends only on the local properties of the scalar field and does not depend on the masses of particles. A decrease in the intensity of fluctuations of particles in a "colder" region means a slowdown in their proper times, i.e. time dilation, while a "thermal" decrease in the distances between them means the contraction of scales.

All these properties are characteristic for gravity, and the fact that they follow from DQM, when the effect of high matter density on the energy density of the scalar field is taken into account, indicates that "thermal" diffusion in an inhomogeneous scalar field represents an analog of gravitation. Since the inhomogeneity arises due to a high concentration of matter, the latter appears as the source of the gravitational field. Thus, gravity in DCM appears as a macroscopic remnant of inhomogeneous quantum fluctuations. In this case, this inhomogeneity arises naturally due to the inverse effect of the presence of a large number of particles on the energy density of the background scalar field.

\section{THERMAL DIFFUSION DERIVATION OF EQUIVALENCE PRINCIPLE AND THE NEWTONIAN FIELD}

A consistent treatment of gravitation requires the relativistic consideration, but some of its basic properties can be studied in the simple case of a weak and static field, when terms of order $1 / c^{2}$ are omitted.

The fluctuation energy of a particle in its rest frame far from massive bodies is equal to its rest energy $E_{0}(\infty)=\tilde{f} \eta=m c^{2}$, where $\tilde{f}=f / \sqrt{2}$. Near a massive body the local rest energy of a particle is $E_{0}(r)=\bar{f} \eta^{\prime}(r)$, which, due to $\eta^{\prime}(r)<\eta$, is less than $E_{0}(\infty)$. It can be expressed through $E_{0}(\infty)$ as:

$$
\begin{aligned}
& E_{0}(r)=\bar{f} \eta^{\prime}(r)=\bar{f} \eta+\bar{f}\left(\eta^{\prime}-\eta\right) \\
& =\bar{f} \eta+\bar{f} \eta\left(\frac{\eta^{\prime}}{\eta}-1\right)=m c^{2}+m \varphi \geq 0,
\end{aligned}
$$

where the function $\varphi(r)$ as some potential is defined as:

$$
\varphi=\frac{\eta^{\prime}}{\eta} c^{2}-c^{2}=c^{\prime 2}-c^{2} \leq 0, \quad \varphi(\infty)=0 .
$$

Here $\varphi(r)$ does not depend on the particle's mass and is determined only by the relation $\eta^{\prime} / \eta$ describing the field.

Thus, the change in the state of the scalar field near a massive body is reduced to the appearance of a potential energy $U(r)$ of particle's interaction with an effective external field $\varphi(r)$. It is important that $U(r)$ is proportional to the particle's mass:

$$
U(r)=m \varphi(r) \leq 0 .
$$

In this case, the potential $\varphi(r)$, according to its definition (4), is negative definite, although the value of the particle's local rest energy, according to (3), remains positive definite $E_{0}(r)>0$.

The Hamilton function of a particle with a drift velocity $\mathbf{v}$ is:

$$
H=m c^{2}+m \varphi+\frac{\mathbf{p}^{2}}{2 m}, \quad \mathbf{p}=m \mathbf{v} .
$$

The equation of motion, following from (6), has the form $\mathbf{a}=-\nabla \varphi$ , where $\mathbf{a}$ is the acceleration. It does not include the particle's mass and, as the result, particles in the effective potential $\varphi(r)$ have the 
same acceleration regardless of their masses. This expresses the principle of equivalence, which distinguishes gravity from other fields, and further, in general relativity, leads to a geometric theory of gravity.

If the field $\varphi$ were some ordinary scalar field, then in the static case its energy density would be written in the form of a positive definite expression $H_{\varphi}=(\nabla \varphi)^{2} / 2 \geq 0$. But, the energy density of the effective field $\varphi$ describes the decrease in the energy density $H_{\phi}(r)$ of the background scalar field $\phi$ around the massive body. For this reason it can be written as the difference between the energy density of $\phi$ at large distance $H_{\phi}(\infty)$ and the local energy density deficit $H_{\varphi}$ :

$$
H_{\phi}(r)=H_{\phi}(\infty)-H_{\varphi}=H_{\phi}(\infty)-\frac{1}{2}(\nabla \varphi)^{2} .
$$

Moreover, the total energy deficit was accumulated in the form of the rest energy of the massive body $M c^{2}$. The constant $H_{\phi}(\infty)$ does not affect to the equations of motion, and the second term in (7) , which is negative definite, manifests itself as energy of the gravitational field in the Newtonian approximation. As in the Newtonian theory, we can normalize the field as $\varphi \rightarrow \varphi / \sqrt{4 \pi G}$, where $G$ is the gravitational constant, and the energy of the scalar field (7) takes the standard form:

$$
H_{\phi}(r)=H_{\phi}(\infty)-H_{\varphi}=H_{\phi}(\infty)-\frac{(\nabla \varphi)^{2}}{8 \pi G} .
$$

Together with the potential energy density $\rho_{m} \varphi$ of particles inside the body, where $\rho_{m}$ is the mass density, this expression gives for $\varphi$ the Poisson equation $\Delta \varphi=4 \pi G \rho_{m}$, the solution of which is the Newtonian potential created by a source $\varphi_{N}$.

Thus, diffusion gravity explains the principle of equivalence and, in the first approximation, reproduces the Newtonian theory of gravity. In this case, the negativity of the energy density of the Newtonian field is naturally explained, since it is not the energy density of an independent «gravitational» field, but is only a local energy deficit of the background scalar field $\phi$, the total energy of which (8) remains positive definite $H_{\phi} \geq 0$.

\section{METRICS INDUCED BY "THERMAL" DIFFUSION IN THE SCALAR FIELD}

The Lagrange function following from $H$ in (6) is:

$$
L=\mathbf{p v}-H=-m c^{2}-m \varphi+\frac{1}{2} m \mathbf{v}^{2}=-m c\left(c+\frac{\varphi}{c}-\frac{1}{2} \frac{\mathbf{v}^{2}}{c}\right) \text { (9) }
$$

Since the action function $S$ in relativity theory is expressed through spacetime interval $d s$, we have:

$$
S=\int d t L=-m c \int d s, \quad d s=d t\left(c+\frac{\varphi}{c}-\frac{1}{2} \frac{\mathbf{v}^{2}}{c}\right)
$$

The square of the expression for $d s$ in (10), neglecting the terms of order $\sim 1 / c^{2}$, gives:

$$
\begin{aligned}
& d s^{2} \simeq\left(1+2 \varphi / c^{2}\right) c^{2} d t^{2}-d \mathbf{r}^{2}=g_{00} c^{2} d t^{2}-d \mathbf{r}^{2}, \\
& g_{00}=1+2 \varphi / c^{2} .
\end{aligned}
$$

Thus, the presence of $\varphi(r)$ changes the time component of the metric $g_{00}$ and, since $\varphi \leq 0$, this leads to dilation of the proper time interval $d \tau$ of a standard clock resting near a massive body with respect to the proper time interval $d t$ of a distant clock:

$$
d \tau=d t g_{00}^{1 / 2}=d t\left(1+2 \varphi / c^{2}\right)^{1 / 2}, \quad d \tau<d t .
$$

This is the well-known gravitational time dilation. The proper time of the distant clock $t$ here is "world time", since its values for simultaneous events are the same everywhere.

As is known from general relativity, from (12) it follows that the conserved energy of a body $E$ is the product of its local energy $E_{0}$ and the time dilation factor $g_{00}^{1 / 2}: E=E_{0} g_{00}^{1 / 2}$. Thermal equilibrium along such a body also satisfies the same condition: $T=T_{0} g_{00}^{1 / 2}$, where $T_{0}$ is the local temperature. The temperature $T$ is the same along the body and in the background scalar field $T_{f} \sim E$ is its analogue, an effective temperature of fluctuations corresponding to the conserved energy $E$. This explains the fact that the local inhomogeneity in the background field does not equalize as it happens in ordinary media, where heat transport and convection equalize the temperatures in the medium and equilibrium occurs. In the scalar field, equilibrium takes place in terms of $T_{f}$, which leads to a new type of equilibrium.

\section{PHYSICAL RESTRICTIONS TO GENERAL RELATIVITY IN DIFFUSION GRAVITY}

The independence of the thermal diffusion acceleration of test particles from their masses leads to the same acceleration of both particles and macroscopic bodies as their collections, including the local frames of reference. But the same acceleration of both objects and frames of reference is indistinguishable from the presence of a nontrivial metric and curvature of spacetime.

The equivalence principle, the basic physical postulate of general relativity, as well as its direct consequence that gravity is a spacetime curvature, now appear as the consequences of thermal diffusion in the scalar field. Therefore, for the transition to the geometric form of gravity theory, it is sufficient to follow the standard general relativistic methods, but be more careful.

General relativity was initially built as extremely general, i.e. as containing not only physical, but a large non-physical part also. In fact, only its physical part, based on the physically realizable frames of reference and their physical coordinates, had experimental confirmation. However, most of the literature on general relativity represents mathematical procedures with frames and their coordinates unrealizable in any physical frame of reference. Diffusion gravity imposes physical restrictions on general relativistic theory of gravity allowing one to preserve a physical part and exclude a non-physical part of the formalism.

Since gravity is represented as a field of deformations of the scalar field, this supposes that all parts of the scalar field in the entire space exist simultaneously, i.e. the field is specified on the hypersurface of simultaneity $t=$ const. of world time $t$.

But a global hypersurface of simultaneity can exist only in a global static frame of reference consisting of mutually resting local 
frames. This means that the gravitational field in the pure form, without kinematic effects, should be described only in a global static frame, preferably in the one where a compact source of this field is rested. The description in all other extended frames with mutually moving local frames leads to mixing of the gravitational and kinematic effects, thus gives a distorted description of gravity. Notice, that the equivalence principle was formulated as the fact that physical phenomena in a local frame moving with acceleration in a region free of gravity occur similarly to ones in a local frame resting in a gravitational field. Diffusion gravity requires to extend the description of the gravitational field in a local resting frame to a global set of resting frames where the scalar field is specified.

One of the methods for describing physical processes in this case is the Rosen's bimetric formalism. In diffusion gravity, the first flat metric of this treatment describes the coordinate effects of the global static frame in a homogeneous scalar field, while the second metric, leading to a nonzero curvature, describes a scalar field in the same static frame, but in the presence of matter, leading to an inhomogeneity of the field. The results of applying this method to diffusion gravity will be discussed elsewhere.

Thus, diffusion gravity, being a field approach, describes gravity on a global static frame. The geometric description turns out to be effective and related only to the specifics of this field, which is not a field in the usual sense, but is only an effective tensor field describing inhomogeneities of the scalar field.

\section{PROBLEMS WITH GRAVITATIONAL ENERGY AND THEIR SOLUTION IN DQM}

In 1864 J.C. Maxwell, in Section «Remarks on the Action of the Force of Gravity» of the treatise «Dynamical Theory of the Electromagnetic Field» [3] noted that if the Newtonian potential is associated with the gravitational field, then the energy density of this field will be negative. Since he considered it unacceptable from the physical point of view, he concluded that this very important fact reveals something about a still unknown physical mechanism of gravitation. Namely, he guessed that a reasonable overcoming of this difficulty can be only if there is a certain medium in entire space with a huge resource of positive energy, greater in magnitude than the deepest of the "gravitational holes". Then the energy density of the gravitational field expresses the local energy density deficit of this medium.

His conclusions were as follows: “...The assumption, therefore, that gravitation arises from the action of the surrounding medium in the way pointed out, leads to the conclusion that every part of this medium possesses, when undisturbed, an enormous intrinsic energy, and that the presence of dense bodies influences the medium so as to diminish this energy wherever there is a resultant attraction. As I am unable to understand in what way a medium can possess such properties, I cannot go any further in this direction in searching for the cause of gravitation" [3].

[1] Zakir, Z. (2021) Quant. and Grav. Phys., 1:013-7610.

[2] Zakir, Z. (2020) Quant. and Grav. Phys., 1:004-7129.

[3] Maxwell, J. K. (1996) A Dynamical Theory of the Electromag. Field, p. 76-77, W.S.P.
As it was shown above, the problem of the negativity of the Newtonian field energy, which caused Maxwell's concern, is naturally solved in diffusion gravity and exactly as Maxwell assumed. Here the desired medium is the fluctuating scalar field having positive energy density, and gravity is the local energy density deficit of this field (7)-(8). This deficit arises at transferring of part of the medium's energy to particles in some region as their rest energy. The resulting inhomogeneity of the energy density of scalar field generates a "thermal" diffusion.

Thus, diffusion gravity explains why "the presence of dense bodies influences the medium so as to diminish this energy wherever there is a resultant attraction" and "in what way the medium can possess such properties".

In general relativity there are some problems with the energy of the gravitational field, in particular, the appearing of pseudotensors instead of the energy-momentum tensor. They are ambiguous and behaved like tensors only under linear transformations. In contrast, in diffusion gravity there is the usual energy-momentum density tensor for the scalar field. If the energy of this field in the presence of matter is distributed inhomogeneously, then this does not lead to problems.

\section{CONCLUSIONS}

Thus, the existence of a background scalar field with a nonzero rms and the assumption that fluctuations of this external field are the source of quantum fluctuations of classical particles leads to DQM without any hypotheses. DQM then reproduces the formalism of quantum mechanics and leads to several consequences.

One of them is thermal diffusion in an inhomogeneous scalar field, which turned out to be the desired physical mechanism of gravitation. This inhomogeneity arises due to the fact that the rest energy (mass) of the particles of a massive body is interpreted as the energy of fluctuations, by the value of which the fluctuation energy of the scalar field has decreased. This circumstance makes the theory of gravity a part of quantum theory, as diffusion gravity, which partly solves the problem of synthesis of theories of these two phenomena. Of the two basic hypotheses of modern physics - quantum fluctuations and gravity - DQM leaves only the first one as a postulate, and the second turns out to be its consequence.

In the present paper main ideas of diffusion gravity and their simple illustrations are presented only. A relativistic formulation of diffusion gravity, the discussion of its consequences for particle physics, astrophysics and cosmology and a review of the literature will be presented in forthcoming publications.

The main tasks for further research in diffusion gravity are the causes and mechanisms of fluctuations of the background field, while the acceptance of these fluctuations as an observational fact leads to a simple and clear physical picture of both quantum and gravitational phenomena, sufficiently simplifying the situation in the foundations of physics. 